\title{
Two Cases of Sclerotic Fibroma of the Skin that Mimicked Keloids Clinically
}

\author{
Mamiko Tosa', Shin-ichi Ansai ${ }^{2}$, Hiroaki Kuwahara ${ }^{3}$, \\ Satoshi Akaishi $^{3}$ and Rei Ogawa ${ }^{1}$ \\ ${ }^{1}$ Department of Plastic, Reconstructive \& Aesthetic Surgery, Nippon Medical School, Tokyo, Japan \\ ${ }^{2}$ Division of Dermatology and Dermatopathology, Nippon Medical School Musashi Kosugi Hospital, Kanagawa, Japan \\ ${ }^{3}$ Department of Plastic and Reconstructive Surgery, Nippon Medical School Musashi Kosugi Hospital, Kanagawa, Japan

\begin{abstract}
Sclerotic fibromas of the skin (SFSs) have a very characteristic histology but the clinical signs are nonspecific. Consequently, it is extremely difficult to make a diagnosis of SFS on the basis of the clinical findings only. We report here two cases of SFS arising on the right scapular region and the right lower leg, respectively. Both cases were clinically similar to keloids. Skin surgeons should remember that SFSs
\end{abstract} \\ are an important differential diagnosis from keloids. (J Nippon Med Sch 2018; 85: 283-286)
}

Key words: sclerotic fibroma, subcutaneous tumors, mimicking keloid

\section{Introduction}

Sclerotic fibromas of the skin (SFSs) are relatively rare fibrous tumors of the skin. Two types of sclerotic fibromas are known to date, namely, the type that presents as multiple lesions and is associated with Cowden's disease ${ }^{1}$, and the type called SFS that presents as solitary lesions in patients without Cowden's disease ${ }^{2}$. SFS is extremely difficult to diagnose on the basis of clinical findings because these findings are usually non-specific; moreover, the incidence of SFS is low. We report here two cases of SFS in the absence of Cowden's disease that clinically mimicked keloids and occurred on the right scapular region and the right lower leg, respectively.

\section{Cases}

\section{Case 1}

A 19-year-old man presented with a tumor on his right scapular region. The tumor had gradually grown over the last 3 years and was slightly itchy. The patient was diagnosed with autism and had severe mental retardation. There was no notable family history or past history regarding the lesion. Doctors at a nearby hospital diagnosed the lesion as being a keloid and the patient was treated with steroid tape for 6 months. However, since the effect of the treatment was poor, he was referred to our hospital. Clinical assessments at the first visit revealed a polypoid $4 \times 4 \times 3 \mathrm{~cm}$-nodule with a broad base that was located on the right scapular region. The lesion was red, waxy in appearance, and had telangiectasia on a smooth surface without adhesion to the underlying tissue (Fig. 1). Surgery under local anesthesia was not possible because the patient's autism meant that he could not stay still long enough for the treatment. Therefore, steroid local injection therapy was initially tried. We first selected triamcinolone local injection therapy, and carried out treatment four times at a frequency of once every two months. Although the tumor surface softened slightly, the injections did not effectively induce tumor shrinkage. The nodule was then surgically excised under general anesthesia and the surrounding skin was sutured.

Histopathology of the resected specimen showed that the tumor was a well-defined hypocellular dermal nodule with an exophytic growth pattern (Fig. 2a). It consisted of thick eosinophilic sclerotic collagen bundles that were separated by clefts and had a storiform pattern (Fig. 2b). The overlying epidermis was flattened. The nuclei of a few fibroblasts were seen among the hyaline collagen bundles (Fig. 2b). These histopathological findings led to a diagnosis of SFS. During the 1-year follow-up af-

Correspondence to Mamiko Tosa, MD, Department of Plastic and Reconstructive Surgery, Nippon Medical School Hospital, 11-5 Sendagi, Bunkyo-ku, Tokyo 113-8603, Japan

E-mail: tosa-m@nms.ac.jp

Journal Website (http://www2.nms.ac.jp/jnms/) 


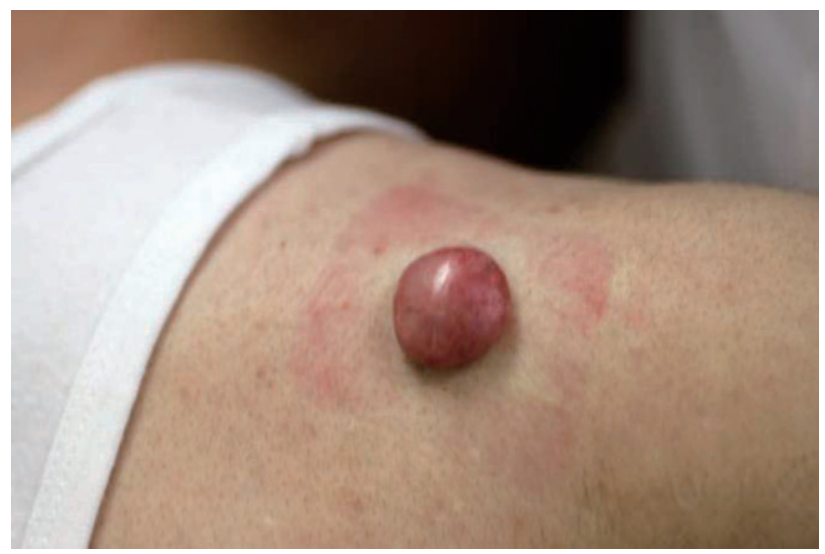

Fig. 1 Clinical findings of Case 1. The lesion was a red polypoid $4 \times 4 \times 3 \mathrm{~cm}$-nodule with a broad base that was located on the right scapular region.
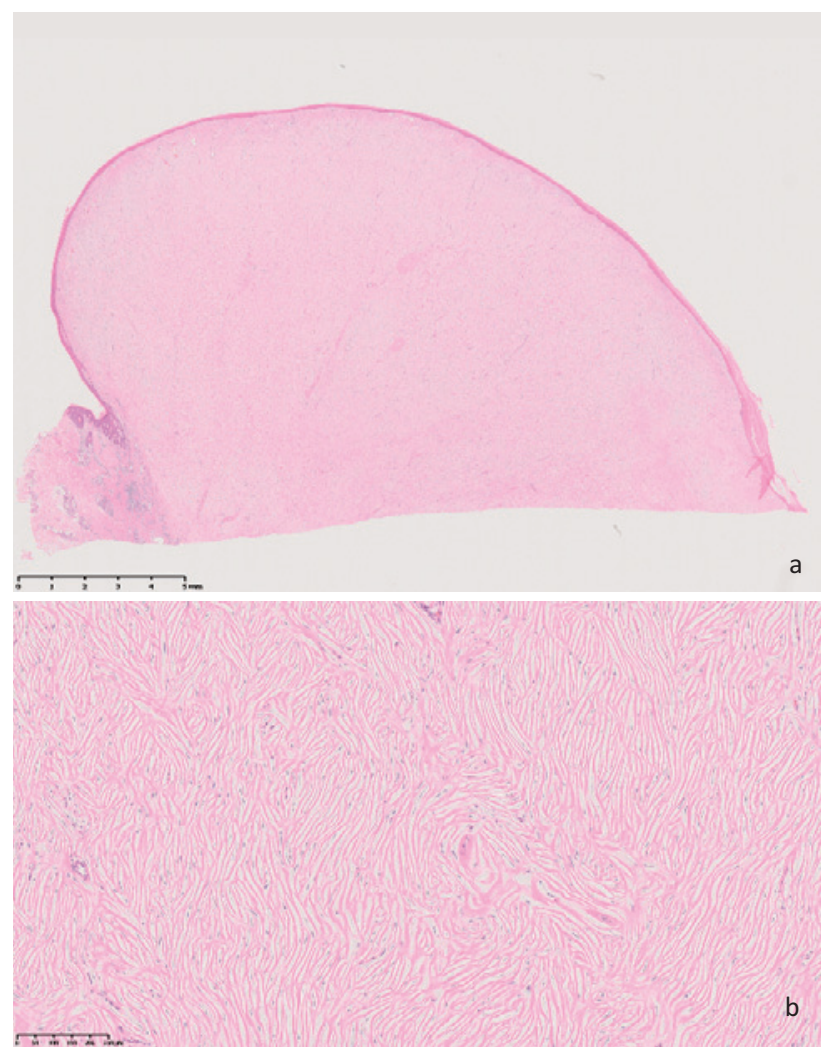

Fig. 2 Histopathological findings of Case 1. (a) The lesion was a well-defined hypocellular dermal nodule that had an exophytic growth pattern. (b) The lesion consisted of thick eosinophilic sclerotic collagen bundles that were separated by clefts and had a storiform pattern. The nuclei of a few fibroblasts can be seen among the hyaline collagen bundles. (H \& E staining)

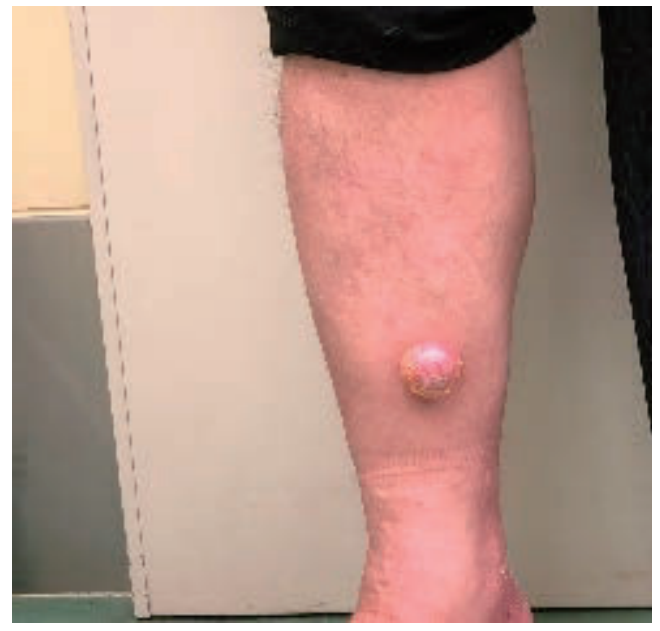

Fig. 3 Clinical findings of Case 2. The lesion was a firm, raised, red polypoid $3 \times 3 \mathrm{~cm}$-skin tumor that was located on the right lower leg.
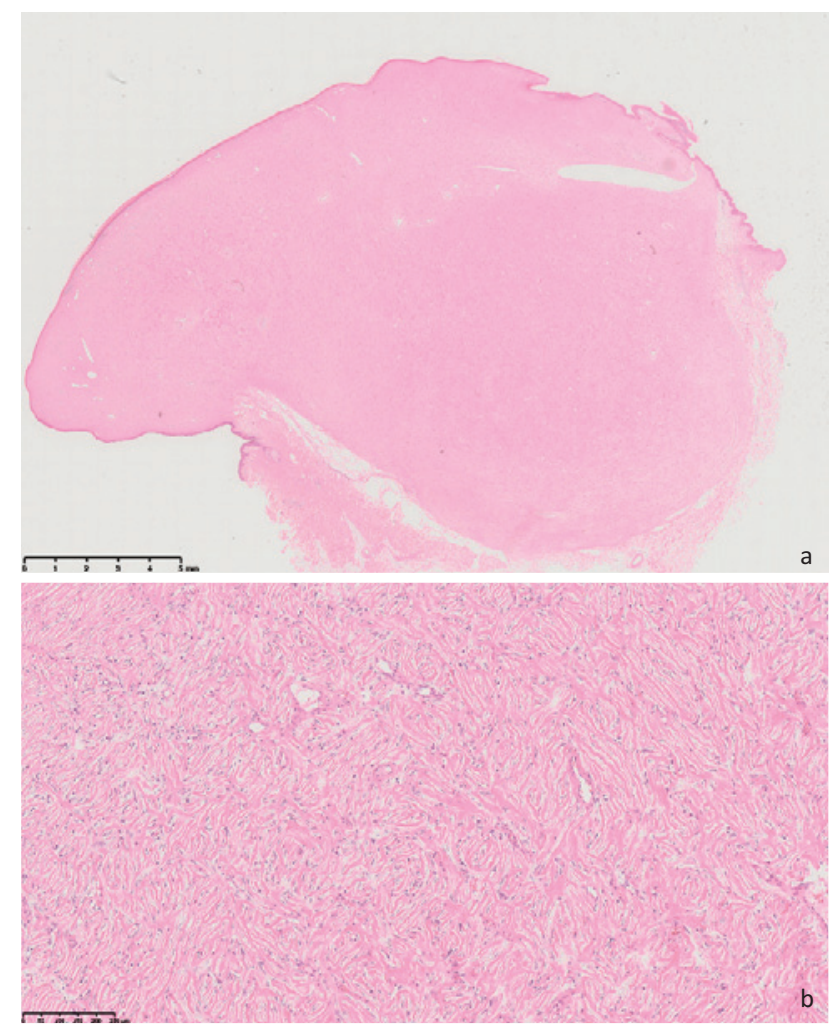

Fig. 4 Histopathological findings of Case 2. (a) The lesion was a well-defined exo- and endophytic nodule in the dermis. (b) The lesion was composed of thick eosinophilic sclerotic collagen bundles that were separated by clefts and had a storiform pattern. There were a few fibroblasts. (H \& E staining) 
Table 1 Summary of all case reports of sclerotic fibromas of the skin in Japan from 1989 to 2017

\begin{tabular}{lccc}
\hline & Case reports in Japan & \multicolumn{2}{c}{ Our cases } \\
& & Case 1 & Case 2 \\
\hline Age of onset (mean) & $18-89(43.2)$ & 19 & 54 \\
Male : Female & $37: 22$ & $\mathrm{M}$ & $\mathrm{M}$ \\
mean size (mm) & 12.5 & 40 & 30 \\
Location & Head and neck 42\% & Scapular region & Lower leg \\
& Extremities 40\% & & \\
\hline
\end{tabular}

ter surgery, the lesion did not recur.

\section{Case 2}

A 54-year-old man presented with a tumor on his right lower leg that had first appeared 30 years ago. It had grown gradually and was painful. An examination revealed it to be an elastic, hard, tender, red polypoid tumor. The lesion was $3 \times 3 \mathrm{~cm}$ in size (Fig. 3). The patient had no notable prior medical problems. His laboratory examinations were normal. The clinical findings suggested that the lesion might be a keloid. However, given the difficulty of making a clinical diagnosis, the lesion was excised under local anesthesia.

The histopathological findings of the resected specimen were almost identical to those of Case 1. Briefly, there was a well-defined exo- and endophytic nodular lesion in the dermis (Fig. 4a), and the lesion was composed of thick eosinophilic sclerotic collagen bundles that were separated by clefts and had a storiform pattern. There were a few fibroblasts (Fig. 4 b).

These findings led to a diagnosis of SFS. The tumor was completely removed by surgery. During the 2 months after surgery, recurrence was not observed.

\section{Discussion}

Sclerotic fibromas were first reported in 1972 as multiple tumors in the tongues of patients with Cowden's disease $^{1}$. Cowden's disease is now known to be an autosomal dominant disorder that is characterized by the growth of multiple hamartomas in multiple organs ${ }^{2}$. It also has several other key diagnostic features, namely, trichlemmoma of the face, palmar keratosis, breast cancer, thyroid cancer, megakaryosis, and Lhermitte-Duclos disease. More minor diagnostic features are lipoma, sclerotic fibroma, goiter, gastrointestinal hamartoma, mental retardation, and chronic mastopathy3. In 1989, similar sclerotic fibromas were reported in patients without Cowden's disease and were called SFSs ${ }^{4}$. Since neither of our two cases satisfied the diagnostic criteria of Cowden's disease, they were therefore diagnosed as having
SFS.

In total, 59 cases of SFS have been reported in Japan between 1989 and 2017. These 59 cases include the two that we report here. The clinical data of these 59 cases are summarized in Table 1. The age at resection was 1889 years (mean: 43.2 years), and the average lesion size at resection was $12.5 \mathrm{~mm}$. There was a male predominance, and the most frequent affected sites were the head and neck and extremities ${ }^{5-7}$ (Table 1). In our cases, the clinical findings were similar to keloids, and in particular, in Case 1, the tumor occurred in the scapular region which is the site of keloids preferentially, so it was difficult to confirm the diagnosis.

The pathogenesis of SFS tumors remains controversial: some believe that SFSs are distinctive benign neoplasms while others suggest that they are a degenerative terminal form of other fibrous tumors such as dermatofibromas. The former opinion is based on the fact that some SFS lesions are immunopositive for proliferating cell nuclear antigen (PCNA) and Ki-67, which are cell proliferation-related proteins, or human type I procollagen, which indicates ongoing collagen synthesis ${ }^{8,9}$. The latter opinion is supported by the findings that dermatofibroma-like tissue is often admixed with SFS tissue $^{10,11}$. The recent report by Chang et al. ${ }^{12}$ suggests that SFSs are associated with inflammatory diseases, especially folliculitis: they tended to share the view that SFSs are a subtype of dermatofibromas. However, it should be noted that, in general, there are differences between dermatofibromas and SFSs. First, dermatofibromas are often accompanied by hyperplastic changes of the epidermis and the boundaries of the lesions are unclear. By contrast, SFSs are not associated with hyperplastic changes of the epidermis and their boundaries are clear. Second, SFSs, but not dermatofibromas, often exhibit expansion into the surrounding tissue. Third, the storiform pattern in SFSs is smaller than that in dermatofibromas. Fourth, SFSs, but not dermatofibromas, are associated with noticeable hyalinization of collagen fibers. 
In relation to the latter histopathological feature, the hyalinized collagen fibers in SFSs somewhat resemble keloidal collagen. However, it is often easy to differentiate them: the hyalinized collagen in keloids is thicker and exhibits a ripple-like arrangement rather than a clear storiform pattern.

In summary, SFSs are relatively rare soft tissue tumors of the skin that show characteristic histopathological findings but non-specific clinical findings. Skin surgeons should include SFSs in the differential diagnosis of keloids or hypertrophic scars.

Conflict of Interest: None declared.

\section{References}

1. Weary PE, Gorlin RJ, Gentry WC Jr, Comer JE, Greer KE: Multiple hamartoma syndrome (Cowden's disease). Arch Dermatol 1972; 106: 682.

2. Tsao H: Update on familial cancer syndromes and the skin. J Am Acad Dermatol 2000; 42: 939-969.

3. Nelen MR, Padberg GW, Peeters EA, Lin AY, van den Helm B, Frants RR, Coulon V, Goldstein AM, van Reen MM, Easton DF, Eeles RA, Hodgsen S, Mulvihill JJ, Murday VA, Tucker MA, Mariman EC, Starink TM, Ponder BA, Ropers HH, Kremer H, Longy M, Eng C: Localization of the gene for Cowden disease to chromosome 10q 22-23. Nat Genet 1996; 13: 114-116.
4. Rapini RP, Golitz LE: Sclerotic fibromas of the skin. J Am Acad Dermatol 1989; 20: 266-271.

5. Abbas O, Ghosn S, Bahhady R, Salman S: Solitary sclerotic fibroma on the scalp of a young girl: reactive sclerosis pattern? J Dermatol 2010; 37: 575-577.

6. Ono F, Mori O, Hashimoto T: An epidermoid cyst with atypical keratinocytes in the lowermost portion of the cyst wall. Kurume Med J 2003; 50: 67-70.

7. Norita T, Akiyoshi E, Kawashima M: Sclerotic fibromas of the skin. J Dermatol 1991; 18: 472-474.

8. Cohen PR, Tschen JA, Abaya-Blas R, Cochran RJ: Recurrent sclerotic fibroma of the skin. Am J Dermatopathol 1999; 21: 571-574.

9. McClement TH: Sclerotic fibroma: a fossil no longer. J Cutan Pathol 1994; 21: 82-85.

10. Pujol RM, de Castro F, Schroeter AL, Su WP: Solitary sclerotic fibroma of the skin: a sclerotic dermatofibroma? Am J Dermatopathol 1996; 18: 620-624.

11. Shitabata PK, Crouch EC, Fitzgibbon JF, Swan-son PE, Adesokan PN, Wick MR: Cutaneous sclerotic fibroma. lmmunohistochemical evidence of a fibroblastic neoplasm with ongoing type 1 collagen synthesis. Am J Dermatopathol 1995; 17: 339-343.

12. Chang SN, Chun SI, Moon TK, Park WH: Solitary sclerotic fibroma of the skin. Degenerated sclerotic change of inflammatory conditions, especially folliculitis. Am J Dermatopathol 2000; 22: 22-25.

(Received, March 13, 2018)

(Accepted, March 27, 2018) 